\section{Energie aus Grünland - eine nachhaltige Entwicklung?}

\author{
von Christine Rösch, ITAS
}

Dauergrünland prägt in vielen Regionen Deutschlands das Landschaftsbild und hat eine herausragende Stellung für den Artenund Biotopschutz, aber auch für die Kulturlandschaft und den Tourismus. Steigende Milchleistungen und der hohe Kostendruck der Milcherzeugung haben in den letzten 10 bis 15 Jahren dazu geführt, dass Grünlandfutter durch kostengünstiges Kraftfutter ersetzt wurde und das Grünland seine Bedeutung als Futterlieferant verloren hat. Auf der Suche nach alternativen Verwendungsmöglichkeiten stellt sich die Frage, ob die Grünlandnutzung zur Erzeugung von Bioenergie eine unter Berücksichtigung von Nachhaltigkeitsaspekten sinnvolle Option darstellt. Mit dieser Fragestellung wird sich ITAS in einem vom Ministerium für Ländlichen Raum und Ernährung des Landes Baden-Württemberg geförderten Projekt „Energie vom Grünland - eine nachhaltige Entwicklung?"“ beschäftigen und dabei das integrative Nachhaltigkeitskonzept der HGF anwenden.
In Deutschland sind knapp $30 \%$ der landwirtschaftlich genutzten Fläche als Dauergrünland ausgewiesen (BMVEL 2003). Grünlandstandorte und ihre Nutzung durch Milchkuh- und Rinderhaltung haben insbesondere in Bayern, Niedersachsen, Baden-Württemberg, Schleswig-Holstein und Nordrhein-Westfalen eine lange Tradition. In diesen Bundesländern finden sich die umfangreichsten Grünlandflächen und die größten Milchvieh- und Rinderbestände Deutschlands (Abb. 1). Die traditionelle Grünlandnutzung ist jedoch seit Jahren rückläufig. Dies betrifft auch Baden-Württemberg. Hier ist die Grünlandfläche seit 1950 auf $44 \%$ ihrer ursprünglichen Größe geschrumpft, aber mit einem Anteil von $39 \%$ an der landwirtschaftlich genutzten Fläche immer noch landschaftsprägend.

Der Rückgang der Grünlandfläche ist im Wesentlichen auf den züchterisch-technischen Fortschritt und den starken Wettbewerbsdruck in der Landwirtschaft zurückzuführen. Die Milch wird bei festgelegten Quoten und steigenden Milchleistungen von immer weniger Kühen erzeugt. Damit wird auch immer weniger Grünland für die Milchviehhaltung benötigt. In Baden-Württemberg verringerte sich die Zahl der Milchkühe innerhalb der letzten 30

Abb. 1: Verteilung des Bestandes an Milchkühen und Rindern in Deutschland nach Bundesländern (Stand: Juli 2003)

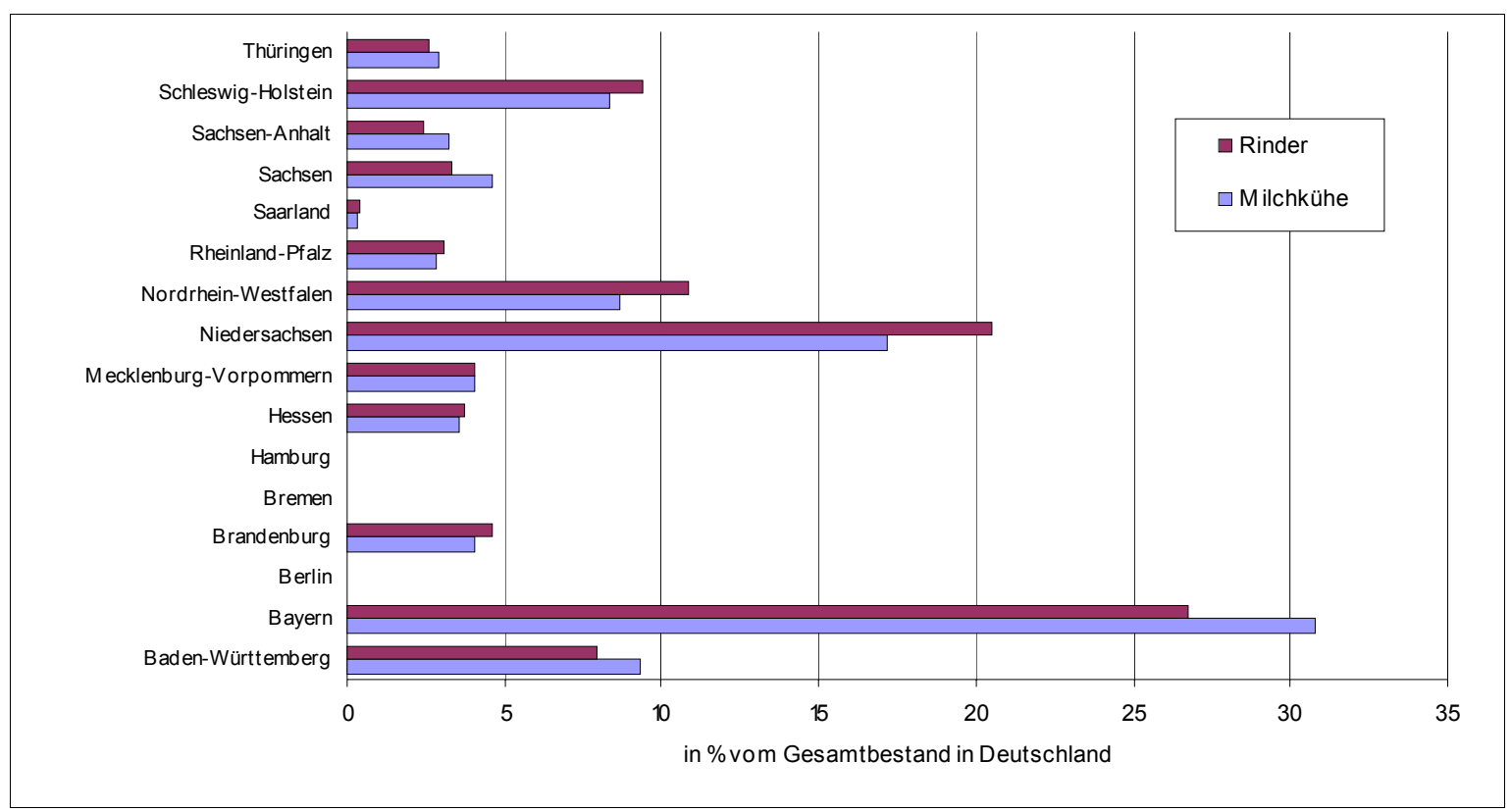


Jahre um $42 \%$. Gleichzeitig ist die Milchleistung pro Kuh auf $6.730 \mathrm{~kg} / \mathrm{Jahr}$ angestiegen. In den nächsten zehn Jahren wird in BadenWürttemberg, bedingt durch die Quotenvorgaben und die bereits über Jahre anhaltende positive Entwicklung der Milchleistung je Kuh, mit einem weiteren Rückgang des Milchviehbestandes von jährlich 2,5 bis $3 \%$ gerechnet.

\section{Hochleistungskühe brauchen immer mehr Kraftfutter}

Eine Trendwende ist nicht absehbar. Im Gegenteil: Der agrarpolitische und ökonomische Druck, die Kosten der Milcherzeugung zu sen$\mathrm{ken}^{1}$ bzw. die Einzeltierleistung weiter zu erhöhen, wird zu einem verstärkten Rückgang der traditionellen Grünlandnutzung führen.

Mit Futter von extensiv bewirtschafteten bzw. nach naturschutzfachlichen Vorgaben genutzten Grünlandflächen ist heute eine wirtschaftliche Milchviehhaltung nicht mehr möglich. Die Grünlandbetriebe in Gunstlagen nutzen deshalb alle produktionstechnischen Möglichkeiten einer intensiven Grünlandfuttererzeugung. Denn nur so können die Anforderungen der Hochleistungskühe an strukturreiches Futter mit hoher Energiedichte erfüllt und hohe Milchleistungen von bis zu $10.000 \mathrm{~kg}$ pro Kuh und Jahr erzielt werden (Buchgraber et al. 2003). Wiesen, die zur Gewinnung von Grassilage $^{2}$ genutzt werden, gehören heute zu den am intensivsten bewirtschafteten landwirtschaftlichen Flächen (Nehls 2002).

Die aus dem hohen Leistungsniveau resultierenden Ansprüche des Milchviehs an die Grundfutterqualität können in der Regel durch intensiv erzeugtes Grünlandfutter allein nicht mehr erfüllt werden. Dies belegt der sinkende Anteil des Grünlandfutters an der Gesamtration bei steigender Milchleistung in Richtung 9.000 bis $10.000 \mathrm{~kg}$ pro Kuh und Jahr (Ernst 2001). Reine Grünlandbetriebe sind deshalb - trotz bester Futterqualität - zunehmend auf den Einsatz von erheblichen Mengen an Kraftfutter ${ }^{3}$ angewiesen (Kühbauch und Anger 1999). Nach Steinwidder (2003a) benötigt eine Kuh mit $5.000 \mathrm{~kg}$ Milchleistung etwa $700 \mathrm{~kg}$ Kraftfutter, eine Kuh mit $8.000 \mathrm{~kg}$ Milchleistung rund $2.000 \mathrm{~kg}$ Kraftfutter pro Jahr neben dem Einsatz von energiereichem Grundfutter.
Die Erzeugung von Kraftfutter wird von der EU-Agrarpolitik bis 2003 durch eine Anbauprämie für Silomais gefördert ${ }^{4}$. Hierdurch wird die wirtschaftliche Attraktivität der Nutzung von Grünland, für dessen Erhalt und Nutzung keine Prämie gewährt wird, weiter verschlechtert.

\section{Folgen der Aufgabe der Grünlandnutzung}

Durch den Einsatz von Mineraldünger und Kraftfutter werden in zunehmendem Umfang Nährstoffe in den Grünlandbetrieb importiert. In den intensiv wirtschaftenden Grünlandbetrieben führt dies zu einem unausgewogenen Verhältnis zwischen Nährstoffinput und Nährstoffoutput (durch die Erzeugung von Milch und Fleisch), mit negativen Folgen für Natur und Umwelt. Von der Aufbringung großer Mengen an mineralischem Stickstoff- und Wirtschaftsdünger besonders betroffen sind die Artenzusammensetzung der Grünlandflächen, deren Vielfalt stark zurückgeht, und das Grundwasser, dessen Qualität sich verschlechtert.

Die Intensivierung der Grünlandnutzung in Gunstlagen geht einher mit der Aufgabe ungünstiger Grünlandstandorte oder deren Umwandlung in Ackerland ${ }^{5}$. Der Prozess der Freisetzung von Grünland infolge der Verdrängung von Grünlandfutter durch Kraftfutter wird sich nicht nur in den Ackerbauregionen weiter fortsetzen (Opitz von Boberfeld 2001). Die Aufgabe der Grünlandnutzung als Rauhfutterfläche infolge einer unbefriedigenden Einkommenssituation trifft vor allem Mittelgebirgsregionen und Grenzertragslagen.

Nicht mehr bewirtschaftete Grünlandflächen, die der natürlichen Sukzession überlassen werden, verbuschen und entwickeln sich allmählich zu Waldbeständen. Mit der Zeit droht die ehemals offene Landschaft langsam zuzuwachsen. In Baden-Württemberg sind von der „Verwaldung“ besonders die Mittelgebirgslagen betroffen. In bestimmten Landkreisen des Schwarzwaldes beträgt der Waldanteil teilweise schon über $90 \%$. Hier würde sich eine weitere Zunahme des Waldes negativ auf das Landschaftsbild und auf den Fremdenverkehr auswirken.

Eine Umwandlung von Grünland in Ackerfläche wäre mit negativen Auswirkungen verbunden, da die positive Wirkung des Grünlands 
als Boden $-{ }^{6}$ und Trinkwasserschutz ${ }^{7}$ wegfallen würde. Grünland ist auch ein wichtiger Bestandteil einer multifunktional ausgerichteten Landwirtschaft und Lebensraum für wild wachsende, lichtbedürftige Pflanzen und frei lebende Tiere $^{8}$. Die Bedeutung des Grünlandes für den Natur- und Umweltschutz in Deutschland lässt sich anhand der Verträge, die im Rahmen von Agrarumweltprogrammen zur Förderung naturund umweltgerechter Bewirtschaftungsformen abgeschlossenen wurden, ablesen. Bei rd. $46 \%$ der Flächen, die im Jahr 2000 unter Vertrag genommen wurden, handelt es sich um Wiesenund Weidenflächen ${ }^{9}$ (BMVEL 2003). Dies entspricht ca. $27 \%$ der Grünlandfläche.

Das Grünland stellt darüber hinaus ein einzigartiges und reizvolles Element der Kultur- und Erholungslandschaft Deutschlands dar und dient als Identifikationsfaktor für die Bürger einer Region.

Es zeigt sich also, dass die Aufrechterhaltung der Nutzung von Grünland eine wichtige Forderung aus der Sicht einer multifunktionalen, nachhaltigen Landwirtschaft darstellt. Bislang konnte das Grünland in Mittelgebirgslagen mit einer angepassten, produktiven Nutzung gepflegt und offen gehalten werden. Trotz der positiven Effekte von Agrarumwelt- und Extensivierungsprogrammen kann das Grünland langfristig jedoch nur dann gesichert werden, wenn es eine neue wirtschaftliche Nutzung erfährt. Die alleinige Pflege des Grünlands und die Entsorgung des Aufwuchses durch die öffentliche Hand bzw. beauftragte Landwirte würde zu sehr hohen Kosten führen, die von der Gesellschaft zu tragen wären. Vor diesem Hintergrund stellt sich die Frage, wie zukunftsfähige Konzepte zur Aufrechterhaltung einer nachhaltigen Grünlandnutzung und zur Offenhaltung der Kulturlandschaft aussehen könnten (Miller 2003).

\section{Sonderformen der Tierhaltung als regio- nale Alternative}

Alternative Verfahren der Tierhaltung auf Grünland, wie die Mutterkuh-, Ochsen-, Ziegen, Schaf-, Pferde- und Wildhaltung wurden bereits in verschiedenen wissenschaftlichen Untersuchungen erforscht (z. B. Ringdorfer 2003, Steinwidder 2003b). Ergebnis der Analysen ist, dass es sich hierbei um Sonderformen der Nutzung handelt, die auf Einzellösungen beschränkt oder nur im Nebenerwerb lukrativ sind. Diese Nutzungsoptionen werden wohl deshalb auch in Zukunft nur eine untergeordnete - wenngleich auch regional wichtige - Rolle einnehmen.

Der Stellenwert der alternativen Tierhaltungsformen hängt (ebenso wie die Bedeutung der o.g. Agrarumweltmaßnahmen) von den agrarpolitischen Rahmenbedingungen und den finanziellen Spielräumen von Bund und Ländern ab. Dies soll nachfolgend am Beispiel der Mutterkuhhaltung illustriert werden. Im Rahmen der Agenda 2000 wurden im Jahr 2002 je Mutterkuh $200 €$ gewährt. Dazu kommt die Extensivierungsprämie von $\mathrm{rd}$. $100 €$, die für eine maximale Besatzdichte von 1,4 Großvieheinheiten ${ }^{10}$ je Hektar Futterfläche gezahlt wird $^{11}$. Ohne Gewährleistung dieser Prämien wäre es nicht möglich, die Mutterkuhhaltung wirtschaftlich $\mathrm{zu}$ betreiben (Steinwidder 2003b). Aufgrund der Quotenzuteilung in der EU sind einer Ausdehnung der prämienberechtigten Mutterkuhhaltung in Deutschland ${ }^{12}$ jedoch enge Grenzen gesetzt. Die Höchstgrenze für Ansprüche auf Mutterkuhprämie wurde für Deutschland auf 639.535 Tiere festgelegt ${ }^{13}$.

\section{Energie aus grüner Biomasse}

Verfahren zur Nutzung des Grünlands außerhalb der tierischen Verwertungsmöglichkeiten sind bislang nur am Rande diskutiert worden. Dabei könnte die Bioenergiegewinnung aus ,frei werdenden" Grünland möglicherweise dazu beitragen, die Verpflichtungen im Rahmen des KyotoVertrages einzuhalten und die Sicherheit der Energieversorgung zu verbessern. Darüber hinaus könnten sich - da die Bereitstellung von Biobrennstoffen relativ personal- und kostenintensiv ist - hierdurch auch positive Effekte auf die Beschäftigung und neue Impulse für den ländlichen Raum einstellen.

Hintergrund dieser Überlegung sind die hohen Ziele, die sich die EU und die Bundesregierung ${ }^{14}$ für den Ausbau der erneuerbaren Energieträger im Allgemeinen und der Biomasse im Besonderen ${ }^{15}$ gesetzt haben (Tab. 1). Baden-Württemberg plant ebenfalls einen höheren Einsatz an Bioenergie. Bis 2010 sollen $2,5 \%$ des Primärenergieverbrauchs in BadenWürttemberg durch dezentrale Nutzung von Biomasse zur Strom- und Wärmeerzeugung gedeckt werden (MUV 2001). 
Tab. 1: Bioenergie in Deutschland: Stand und Ziele

\begin{tabular}{|l|r|r|}
\hline & 2000 & \multicolumn{1}{c|}{2010} \\
\hline Biokraftstoffe & $0,8 \%$ & $5,75 \%$ \\
\hline Biostrom & $0,3 \%$ & steigend* \\
\hline Biowärme & $3,7 \%$ & steigend \\
\hline $\begin{array}{l}\text { Bioenergie am Pri- } \\
\text { märenergieverbrauch }\end{array}$ & $1,78 \%$ & steigend \\
\hline
\end{tabular}

* $\quad 12,5 \%$ für Regenerative Energieträger insg.

Die Ausweitung der energetischen Nutzung von Biomasse auf das Grünland sollte - darüber sind sich Politik und Gesellschaft weitgehend einig auf eine möglichst nachhaltige Weise erfolgen.

\section{Zielsetzung des neuen ITAS-Projekts}

Vor dem Hintergrund einerseits der ,frei werdenden" Grünlandflächen und andererseits dem Ziel, den Anteil der Biomasse an der Energieversorgung zu erhöhen, stellt sich die Frage, welche Stellung die energetische Nutzung von Grünland im Kontext einer nachhaltigen Entwicklung einnehmen könnte. ITAS ist vom Ministerium für Ländlichen Raum und Ernährung des Landes Baden-Württemberg beauftragt worden, diese Fragestellung am Beispiel Baden-Württemberg zu bearbeiten.

In dem Projekt „Energie vom Grünland eine nachhaltige Entwicklung?" soll zunächst der Stand der „Freisetzung“ von Grünlandflächen aus der Futterproduktion in BadenWürttemberg erfasst und räumlich differenziert dargestellt werden. Die Regionen mit einem hohen Anteil an nicht mehr für die Tierernährung genutztem Grünland sollen identifiziert und charakterisiert werden.

Aufbauend auf dem regional aufgefächerten Ausgangsbestand an Grünlandflächen sollen Annahmen über die mittel- bis langfristige Entwicklung und Allokation der Rind- und Milchviehhaltung und die zukünftige Nutzung des Grünlands zur Rauhfuttergewinnung ausgearbeitet werden. Neben eigenem Erfahrungsund Fachwissen (Rösch et al. 2002) sollen für diesen Arbeitsschritt die vorhandene Statistik und Fachliteratur sowie elektronische Informationsquellen ausgewertet werden. Bei der Bearbeitung der zukünftigen Entwicklung der
Grünlandnutzung wird davon ausgegangen, dass gerade die Experten im Bereich Grünland am ehesten in der Lage sind, eine fundierte Bewertung der Perspektiven der Grünlandnutzung vorzunehmen. Daher sollen mit verschiedenen Fachleuten Tiefeninterviews durchgeführt werden, um einen vollständigen Informationsüberblick zu erhalten. Die Regionen, die von der zukünftigen "Grünlandfreisetzung“ besonders betroffen sein werden, sollen herausgefiltert und klassifiziert werden.

Im zweiten Teil der Arbeit sollen auf der Basis des in verschiedenen Projekten gewonnenen Fachwissens (Rösch 1998, 1999, 2000, 2001, Leible et al. 2003) sowie neuster Literatur die wesentlichen technischen, ökonomischen und ökologischen Kenngrößen ausgewählter Verfahren zur Energiegewinnung aus Grünland erarbeitet und dargestellt werden. Darauf aufbauend werden Prozesskettenanalysen für ausgewählte Verfahren durchgeführt. Zu den Verfahren, die näher betrachtet werden, gehören die Vergärung in Biogasanlagen und die Mitverbrennung in Biomasseheiz(kraft)werken bzw. Kohlekraftwerken. Nicht in die Betrachtung einbezogen werden soll die Herstellung von Biokraftstoffen aus Grünland. Für die ausgewählten Verfahren soll der aktuelle Stand des Wissens in Forschung, Entwicklung, Demonstration und Kommerzialisierung ermittelt und beschrieben werden.

Eine detaillierte Analyse einerseits des Umfangs an „frei werdendem“ Grünland und andererseits des Anforderungsspektrums der energetischen Verwertungstechniken soll zeigen, welche Verfahren prinzipiell zur Nutzung des Grünlandaufwuchses geeignet wären.

\section{Anwendung des integrativen Konzepts}

Zur Beurteilung der Nachhaltigkeit der ausgewählten Verfahren zur Energiegewinnung aus Grünland wird das im HGF-Projekt erarbeitete integrative Nachhaltigkeitskonzept (Kopfmüller et al. 2001) herangezogen. Anhand der konkretisierten Mindestanforderungen, die erfüllt werden müssen, um eine nachhaltige Entwicklung zu erreichen, sollen die Indikatoren, Ziele und Strategien einer nachhaltigen Nutzung des „frei werdenden“ Grünlands erarbeitet werden.

Die Anwendung des integrativen Konzepts als Instrument zur Bewertung der Nachhaltigkeit 
energetischer Nutzungsverfahren hat gegenüber anderen Methoden, wie z. B. der Ökobilanz, den Vorteil, das sowohl ökologische als auch ökonomische und soziale Belange integrativ betrachtet werden. Auf diese Weise werden für das Vorhaben interessante Aspekte, wie z. B. die selbständige Existenzsicherung in der Landwirtschaft, die gerechte Verteilung der Umweltnutzungsmöglichkeiten, der Erhalt der kulturellen Funktionen der Natur und die Partizipation von Landwirten an gesellschaftlichen Entwicklungsprozessen in die Analyse und Bewertung einbezogen. Institutionelle Aspekte, wie sie in den Regeln zur Internalisierung externer Kosten und zur internationalen Zusammenarbeit angesprochen sind, spielen ebenfalls eine Rolle.

Um das integrative Konzept auf die Fragestellung des Grünlandprojektes anwenden zu können, muss es entsprechend zugeschnitten werden. Mindestanforderungen, deren Sachverhalte den Untersuchungsgegenstand nicht betreffen bzw. nur am Rande tangieren, werden in der Arbeit nicht behandelt. Die Anzahl der Indikatoren, die in der Arbeit betrachtet werden, ist aus Gründen der Bearbeitbarkeit ebenfalls zu begrenzen. Durch eine ausgewogene Auswahl der Indikatoren soll jedoch gewährleistet werden, dass - trotz Verwendung eines reduzierten Indikatorensatzes - der integrative Nachhaltigkeitsansatz erhalten bleibt und die Indikatorenauswahl keinen Bias bedingt.

Außerdem muss der integrative Ansatz teilweise stärker disaggregiert und konkretisiert werden, um die nachhaltigkeitsrelevanten Aspekte der energetischen Grünlandnutzungsoptionen umfassend vergleichen und beurteilen zu können. Eine kleinräumige Betrachtungsweise ist auch deshalb wichtig, weil hierdurch die speziellen Gegebenheiten (Stärken und Schwächen) der ausgewählten Regionen eine stärkere Berücksichtigung finden.

\section{Regionale Betrachtung}

Das HGF-Projekt hat das globale Nachhaltigkeitsleitbild in nationale Nachhaltigkeitsziele, -indikatoren und -strategien umgesetzt. Auf der Landesebene hat das Land Baden-Württemberg im Rahmen der Agenda 21 einen Umweltplan (MUV 2001) entwickelt, der einen langfristigen, auf das Jahr 2010 ausgerichteten Orientierungsrahmen und Zielvorstellungen darüber enthält, wie eine dauerhaft-umweltgerechte Entwicklung des Landes Baden-Württemberg aussehen und verwirklicht werden kann. Dabei besteht eine enge Verknüpfung mit dem Landesentwicklungsplan, der im Rahmen seiner Fortschreibung ebenfalls verstärkt am Prinzip der Nachhaltigkeit ausgerichtet wurde. Im Rahmen des Projekts soll das Nachhaltigkeitsleitbild auf die regionale Ebene heruntergebrochen und angewandt werden. Es ist vorgesehen, in zwei noch auszuwählenden Regionen Baden-Württembergs eine tiefer gehende Nachhaltigkeitsbetrachtung vorzunehmen. Die Unterschiede in der naturräumlichen, wirtschaftlichen und sozialen Ausstattung der Regionen könnten - so die Ausgangshypothese - einen wesentlichen Einfluss auf die Ergebnisse der Arbeit haben. Eine regionalspezifische Betrachtung ist im Rahmen des Projektes auch deshalb von Bedeutung, da zentrale Anlagen zur Verwertung der Energie aus Grünland aufgrund der damit verbundenen hohen Transportkosten ${ }^{16}$ kaum wirtschaftlich sind.

Darüber hinaus stellen sich bei regionaler Betrachtungsweise bestimmte Nachhaltigkeitsaspekte anders dar, als auf einer hochaggregierten Bundes- oder Landesebene. Ein Beispiel: Die verstärkte energetische Nutzung von (grüner) Biomasse wird in Deutschland vor allem mit dem Ziel, die klimarelevanten Emissionen $\mathrm{zu}$ reduzieren, gefördert. In ländlichen (und strukturschwachen) Regionen stellt die traditionelle Grünlandnutzung zur Futterbereitstellung dagegen eine wesentliche Grundlage für Einkommen und wirtschaftliche Entwicklung dar. Das regionale Management des Grünlands, das sowohl ökologische als auch ökonomische und soziale Aspekte berücksichtigt, bildet dort den wichtigsten Gestaltungsraum für eine nachhaltige Entwicklung. Im Projekt ist deshalb vorgesehen, zwei Regionen in Baden-Württemberg näher zu untersuchen, die von der „Grünlandfreisetzung" besonders betroffen sind.

Nachhaltige Entwicklung ist (auch) durch einen gesellschaftspolitischen Aushandlungsprozess gekennzeichnet. Um diesen Diskurs in Bezug auf die Frage der zukünftigen Grünlandnutzung in Gang zu setzen, sollen die im Rahmen des Projektes erzielten Ergebnisse zur nachhaltigen energetischen Nutzung von Grünlandflächen mit den Akteuren der ausgewählten Regionen (Landwirte, Verbandsvertreter, kom 
munale Vertreter, Tourismusindustrie) diskutiert werden.

\section{Zusammenfassung}

Ziel des Vorhabens ist es, konkrete modellhafte Lösungskonzepte und Maßnahmen für das ,frei werdende" Grünland zu entwickeln, auf deren Grundlage gleichermaßen ökologische, ökonomische und soziale Zielsetzungen verwirklicht werden können. Zur Bearbeitung des Themas soll der im Rahmen des HGF-Projektes entwikkelte integrative Nachhaltigkeitsansatz (Coenen und Grunwald 2003) eingesetzt werden. Im Vergleich zum HGF-Projekt fokussiert das Vorhaben stärker auf die Zusammenführung und kohärente Betrachtung der Bereiche Energie und Landwirtschaft.. Die Anwendung des integrativen Nachhaltigkeitskonzeptes auf eine konkrete Problemstellung in "Praxisregionen“ mit dem Ziel, Entscheidungswissen für eine nachhaltige Entwicklung des Grünlands zu erarbeiten, ist bislang noch nicht durchgeführt worden und stellt eine große Herausforderung dar.

\section{Anmerkungen}

1) Die am 26. Juni 2003 vom Luxemburger Agrarrat beschlossene Reform der Gemeinsamen Agrarpolitik (GAP) beinhaltet die über die Agenda 2000 hinaus gehende Senkung des Milchpreises von 2004 bis 2008 um ca. $26 \%$ von 27,7 auf 20,8 Ct/kg. Die Preissenkungen werden mit $1,181 \mathrm{Ct} / \mathrm{kg}$ in $2004,2,365 \mathrm{Ct} / \mathrm{kg}$ in 2005, 3,55 Ct/kg ab 2006 ausgeglichen. Das Quotensystem wird bis 2014/15 verlängert. Im Jahr 2007 soll die Milchquote um 1,5 \% (Basis: 1999) angehoben werden, was zu einer weiteren Erhöhung des Preisdrucks auf Milch führen wird. Die mit dieser Reform verbundenen Einkommensverluste (ohne Modulation) in Deutschland werden auf rd. $1 \mathrm{Mrd}$ € geschätzt (DBV 2003). Um sie mit einer Grünlandprämie auszugleichen, wären rd. $300 € /$ ha erforderlich.

2) Grassilage bringt höhere Erträge als Heu, kann weniger wetterabhängig geborgen werden und ist von daher die derzeit wirtschaftlichste Form der Grünlandnutzung.

3) Unter langfristigen (Voll-)Kostenbedingungen ist Kraftfutter oder Mais preiswerter als Grünlandaufwuchs. Grassilage kostet je Energieeinheit $\mathrm{rd}$. 1,3 Ct/MJ Netto-Energie Laktation (NEL), Maissilage dagegen nur rd. 1,1 Ct/MJ NEL (Stark 2002).
4) Gemäß dem Beschluss der europäischen Agrarminister zur EU-Agrarreform im Juni 2003 werden die Direktzahlungen von der Produktion entkoppelt. Auf dieser Basis wird zukünftig die Aufhebung der Diskriminierung von Grünland beispielsweise durch die Einführung einer regional einheitlichen Acker- und Grünlandprämie möglich. Mit der Bindung der Prämienzahlung an Umweltauflagen würde zudem ein Signal gegen die Intensivierung der Landwirtschaft gesetzt.

5) Die im novellierten Bundesnaturschutzgesetz definierte „gute fachliche Praxis“ verbietet den Grünlandumbruch lediglich auf erosionsgefährdeten Hängen, auf Standorten mit hohem Grundwasserstand und auf Moorstandorten.

6) Grünland verhindert auf hängigen Flächen die Bodenerosion und den Eintrag von nährstoffreichem Erosionsmaterial in Oberflächengewässer.

7) Auch bei intensiver Grünlandnutzung beträgt der Nitratgehalt im Bodenwasser nur einen Bruchteil des für Trinkwasser geltenden Richtbzw. Grenzwertes. Deshalb liegen viele Wasserschutzgebiete unter Grünland.

8) Infolge der Intensivierung der Grünlandnutzung sind heute viele Vogelarten, die auf Grünland brüten (z.B. Kiebitz und Feldlerche) oder dort ihre Nahrung suchen (z.B. Weißstorch), auf dem Rückzug.

9) Etwa ein Drittel der Grünlandverträge sind mit speziellen naturschutzorientierten Auflagen verbunden. Der überwiegende Teil dient einer generellen Extensivierung der Nutzung.

10) Der Begriff Großvieheinheit (GVE) wird verwendet, um Tiere verschiedener Art und mit unterschiedlichem Gewicht für rechnerische Zwecke miteinander vergleichbar zu machen. Eine GVE entspricht dem Lebendgewicht einer Kuh $(500 \mathrm{~kg})$.

11) Die Futterfläche für die Extensivierungsprämie muss zu mindestens $50 \%$ aus Weideland bestehen.

12) Die Mutterkuhhaltung ist in Deutschland regional unterschiedlich verteilt: $41 \%$ der Mutterkühe werden in Ostdeutschland (v. a. Brandenburg und Mecklenburg-Vorpommern) gehalten (Hundt 2002).

13) Die Prämienrechte für Mutterkühe belaufen sich in der EU auf insgesamt rd. 10,8 Mio. Stück. Deutschland hält mit knapp 0,64 Mio. Tieren lediglich $6 \%$ dieser Ansprüche. Der französische Anteil beträgt hingegen mit rd. 3,77 Mio. Tieren immerhin $35 \%$ der Gesamtquote der EU.

14) Die Bundesregierung hat den Anteil regenerativer Energien als Indikator für nachhaltige Entwicklung ausgewählt (Bundesregierung 2001). 
15) Der Biomasse kommt bei der Erhöhung des Anteils regenerativer Energieträger an der Energieversorgung aufgrund ihrer noch unausgeschöpften Potenziale eine besondere Bedeutung zu.

16) Dies hängt zum einen mit der geringen Menge sowie dem dezentralen Anfall des Grünlandaufwuchses und zum anderen mit der relativ niedrigen Transportdichte der grünen Biomasse zusammen.

\section{Literatur}

BMVEL - Bundesministerium für Verbraucherschutz, Ernährung und Landwirtschaft, 2003: Ernährungs- und agrarpolitischer Bericht der Bundesregierung. Berlin: BMVEL

Buchgraber, K.; Resch, R.; Blaschka, A., 2003: Entwicklung, Produktivität und Perspektiven der österreichischen Grünlandwirtschaft. 9. Alpenländisches Expertenforum, 27.-28.03.2003. Bundesanstalt für alpenländische Landwirtschaft (BAL), Gumpenstein, Österreich, S. 9-17

Bundesregierung, 2001: Perspektiven für Deutschland. Unsere Strategie für eine nachhaltige Entwicklung, 17. April 2002; http://www.nachhaltigkeitsrat. de/service/download/pdf/Nachhaltigkeitsstrategie Kurzfassung.pdf

Coenen, R., Grunwald, A. (Hrsg.), 2003: Nachhaltigkeitsprobleme in Deutschland - Analyse und Lösungsstrategien. Berlin: edition sigma (Global zukunftsfähige Entwicklung - Perspektiven für Deutschland, Band 5)

DBV - Deutscher Bauernverband, 2003: Die Auswirkungen der Reform. Pressemitteilung vom 26.06.2003; http://www.bauernverband.de/konkret_ 699.html

Ernst, P., 2001: Grünlandmanagement für Hochleistungstiere aus pflanzenbaulicher Sicht. Arbeitsunterlagen zur DLG-Grünlandtagung 2001: Nachhaltige Futterproduktion aus dem Grünland. DLG, Frankfurt a.M., S. 13-16

Hundt, M., 2002: Mutterkuhhaltung - ein wichtiger Markt. ZMP-Zentralbericht vom 12.12.2002. Bonn, S. 2-3

Kopfmüller J.; Brandl, V.; Jörissen, J.; Paetau, M.; Banse, G.; Coenen, R.; Grunwald, A., 2001: Nachhaltige Entwicklung integrativ betrachtet. Konstitutive Elemente, Regeln, Indikatoren. Berlin: edition sigma (Global zukunftsfähige Entwicklung - Perspektiven für Deutschland, Bd. 1)

Kühbauch, W.; Anger, M., 1999: Modellrechnungen zur Senkung von Stickstoffverlusten und Nährstoffüberschüssen im Milchviehbetrieb auf Grünland. Sonderdruck aus Agrarbiological Research,
Band 52, Heft 1, Darmstadt: VDLUFA-Verlag, S. 77-84

Leible, L.; Arlt, A.; Fürniß, B.; Kälber, S.; Kappler, G.; Lange, S.; Nieke, E.; Rösch, Ch.; Wintzer, D., 2003: Energie aus biogenen Rest- und Abfallstoffen. Bereitstellung und energetische Nutzung organischer Rest- und Abfallstoffe sowie Nebenprodukte als Einkommensalternative für die Land- und Forstwirtschaft - Möglichkeiten, Chancen und Ziele. Forschungszentrum Karlsruhe, Wissenschaftliche Berichte, FZKA 6882

Miller, J., 2003: Pressekonferenz zur Grünlandbewirtschaftung in Bayern am 14.07.2003 am Spitalhof in Kempten. Manuskriptfassung; http:/www.stmlf. bayern.de/publikationen/ministerreden/2003/ re074-03.html

MUV - Ministerium für Umwelt und Verkehr BadenWürttemberg, 2001: Umweltplan Baden-Württemberg; http://www.uvm.baden-wuerttemberg.de/abt2/ umweltplan/text/umpl01.htm

Nehls, G., 2002: Grünlandnutzung und Naturschutz - die Flächenprämie bringt's zusammen. Der Kritische Agrarbericht 2002. Hamm: ABL Bauernblatt Verlags-GmbH, S. 6-12

Opitz von Boberfeld, W., 2001: Grünlandumwidmung von konventioneller Milch- zur ökologischen Fleischerzeugung. Arbeitsunterlagen zur DLGGrünlandtagung 2001: Nachhaltige Futterproduktion aus dem Grünland. DLG, Frankfurt a.M., S. 27-36

Ringdorfer, F., 2003: Nachhaltige Nutzung des Grünlands mit Schafen und Ziegen. 9. Alpenländisches Expertenforum, 27.-28.03.2003. Bundesanstalt für alpenländische Landwirtschaft (BAL), Gumpenstein, Österreich, S. 69-71

Rösch, C., 1998: Verfahren zur energetischen Nutzung von Biomasse mit Brennstoffzellen - Grundlagen und Systeme. In: Fachagentur Nachwachsende Rohstoffe e.V. (Hrsg.): Energetische Nutzung von Biomasse mit Brennstoffzellenverfahren. Gülzower Fachgespräche, Gülzow, S. 7-33

Rösch, C., 1999: Conclusions of the Country Reports "Standardisation of Solid Biofuels in the EU" - Results of the FAIR-Project CT98-3952; DG XIISSMI. Proceedings of the 2nd Joint Meeting with CEN/BT/WG 108 Solid Biofuels and the partners of the FAIR and THERMIE Consortias, 14th to 15th of September 1999, Stockholm, S. 6-10

Rösch, C., 2000: Technical and non-technical barriers of co-combustion of biomass in Germany. In: NOVEM (ed.): Report of the second seminar on addressing the constraints for successful replication of demonstrated technologies for co-combustion of biomass/waste from 22-11 to 23-11-99 in Geertruidenberg, Netherlands 
Rösch, C., 2001: Nachhaltige Nutzung von Biomasse als Energieträger. In: TA-Datenbank-Nachrichten, 10. Jg., Heft 3, S. 27-34

Rösch, C.; Backhaus, R.; Meyer, B.C., 2002: Nachhaltige Landwirtschaft in Betrieb und Landschaft. In: Grunwald, A. (Hrsg.): Technikgestaltung für eine nachhaltige Entwicklung. Von der Konzeption zur Umsetzung. Berlin: edition sigma (Global zukunftsfähige Entwicklung - Perspektiven für Deutschland, Bd. 4), S. 209-244

Stark, G., 2002: Rechnet sich Qualitätsfutter vom Grünland? Vortrag auf der DLG-Wintertagung; http://presse.dlg.org/workspace/de/page23067.html

Statistisches Bundesamt, 2003: Viehwirtschaft in Deutschland differenziert nach Bundesländern; http://www.destatis.de/jahrbuch/jahrtab22.htm

Steinwidder, A., 2003a: Persönliche Mitteilung. BAL Gumpenstein, Abteilung für Produktions- und Nutztierverfahren, A-8952 Irdning

Steinwidder, A., 2003b: Extensive Produktionsalternativen im Grünland mit Rindern. 9. Alpenländisches Expertenforum, 27.-28.03.2003. Bundesanstalt für alpenländische Landwirtschaft (BAL), Gumpenstein, Österreich, S. 63-68

\section{Kontakt}

Dr. Christine Rösch

Forschungszentrum Karlsruhe $\mathrm{GmbH}$

Institut für Technikfolgenabschätzung und Systemanalyse (ITAS)

Postfach 3640, 76021 Karlsruhe

Tel.: +49 (0) 7247 / 82 - 2704

Fax: +49 (0) 7247 / 82 - 4806

E-Mail: roesch@itas.fzk.de

Internet: http://www.itas.fzk.de

》)

\section{Entwicklung eines Nachhaltig- keitsindexes für börsennotierte Unternehmen}

\author{
von Reinhard Paulesich, Wirtschaftsuni- \\ versität Wien
}

Im Rahmen eines Forschungsprojekts „Ecological and Social Efficiency (EASEY)“ wird gegenwärtig von der Abteilung für Wirtschaft und Umwelt des Instituts für Wirtschaftsgeographie, Regionalentwicklung und Umweltwirtschaft der Wirtschaftsuniversität Wien in Zusammenarbeit mit weiteren Partnern aus Wissenschaft und Wirtschaft sowie der Wiener Börse AG ein Nachhaltigkeitsindex für Unternehmen im Prime Market Segment der Wiener Börse entwickelt, dessen Einführung im Jahr 2004 vorgesehen ist. Ziel ist es, die gängige Unternehmensbewertung nach Kriterien wie Kapitalrentabilität und Gewinnerwartungen um Aspekte der Nachhaltigkeitsleistung von Unternehmen in ökologischer und sozialer Hinsicht zu erweitern.

Das Forschungsprojekt „Ecological and Social Efficiency (EASEY)“ wird vom österreichischen Bundesministerium für Verkehr, Innovation und Technologie (BMVIT) im Rahmen des Forschungsprogramms „Fabrik der Zukunft" gefördert. Die Leitung des Projekts hat die Abteilung für Wirtschaft und Umwelt des Instituts für Wirtschaftsgeographie, Regionalentwicklung und Umweltwirtschaft der Wirtschaftsuniversität Wien in Zusammenarbeit mit der Unternehmensberatung Wien, dem Zentrum für Soziale Innovation Wien, dem Forschungsinstitut für Nachhaltiges Wirtschaften der Universität Innsbruck, der Firma PriceWaterhouseCoopers und der Wiener Börse AG.

Die Bearbeitung des Projekts erfolgt in mehreren Phasen (siehe Abb. 1). Zunächst wurden bestehende Bewertungskonzepte (z. B. Dow Jones Sustainability Group Index) einer SWOTAnalyse (Strengths-Weaknesses-OpportunitiesThreats) unterzogen und Experten-Workshops zur Thematik durchgeführt. Darauf aufbauend wurde ein Set von Nachhaltigkeitsindikatoren für Unternehmen erarbeitet. Solche Indikatoren müssen in einen Zusammenhang gestellt werden, wenn die Werte, die sie transportieren, für eine wissenschaftliche Argumentation brauchbar sein sollen. Das EASEY Indikatorenset steht 